\title{
Die Bindungswirkung von Stellungnahmen des Deutschen Bundestages im Rahmen der Zusammenarbeit mit der Bundes- regierung in EU-Angelegenheiten
}

\author{
Richard Wichmann
}

Die Übertragung von Hoheitsrechten auf die Europäische Union nach Art. 23 I 2, 3 GG findet ihre Legitimation im Demokratieprinzip der Art. 20 und 38 GG und darf daher nur durch den unmittelbar durch das Volk legitimierten Bundestag erfolgen. Eine eigenständige Entscheidungsmacht steht der Bundesregierung in diesem Bereich nicht zu. Hierfür bedarf sie der für sie rechtlich verbindlichen, vorherigen Ermächtigung des Bundestages. Darüber hinaus hat das Bundesverfassungsgericht (BVerfG) in seinem Lissabon-Urteil ${ }^{1}$ keine vorrangige Entscheidungskompetenz des Bundestages in EU-Angelegenheiten festgeschrieben. Die sich an dem Urteil orientierende erneuerte Begleitgesetzgebung ${ }^{2}$ komplettiert zwingende Mitwirkungserfordernisse von Bundestag und Bundesrat daher auch nur in Bezug auf mögliche Machtverschiebungen von der nationalen auf die europäische Ebene. Die im EUZBBG $^{3}$ geregelten Stellungnahmen stehen demgegenüber im Ermessen des Bundestages. Einer zwingenden Mitwirkung bedarf es hier nicht, denn sollte das Vorhaben auf das sich das Stellungnahmerecht bezieht, eine Kompetenzerweiterung der EU ermöglichen ${ }^{4}$, greifen die Mitwirkungserfordernisse des IntVG. ${ }^{5}$

Im Zuge der durch das Lissabon-Urteil nötig gewordenen Erneuerung der Begleitgesetzgebung kam es erneut zu Diskussionen über die Notwendigkeit, dem Bundestag in Angelegenheiten der EU ein Weisungsrecht gegenüber der Bundesregierung einzuräumen. Bestrebungen in diese Richtung gab es insbesondere seitens der Fraktion Die Linke ${ }^{6}$ und der CSU. ${ }^{7}$

1 Urteil vom 30. Juni 2009, in: NJW, 62. Jg. (2009), H. 31, S. 2267.

2 Gesetz über die Wahrnehmung der Integrationsverantwortung des Bundestages und des Bundesrates in Angelegenheiten der EU (IntVG) vom 22. September 2009, zuletzt geändert durch Gesetz vom 1. Dezember 2009, BGBl. I, S. 3822; Gesetz über die Zusammenarbeit von Bund und Ländern in Angelegenheiten der EU vom 12. März 1993, zuletzt geändert durch Gesetz vom 22. September 2009, BGBl. I, S. 3031; Gesetz über die Zusammenarbeit von Bundesregierung und Deutschem Bundestag in Angelegenheiten der EU (EUZBBG) vom 12. März 1993, zuletzt geändert durch Gesetz vom 22. September 2009, BGBl. I, S. 3026.

3 Vgl. ebenda.

4 Vgl. \$3 II EUZBBG.

5 Siehe Fußnote 2.

6 Siehe Gesetzentwurf zur Änderung des Grundgesetzes, BT-Drs. 16/13928, S. 3: „Artikel 23 Absatz 3 Satz 2 wird wie folgt geändert: Die Bundesregierung ist an diese Stellungnahmen des Bundestages gebunden."

7 Entwurf eines Gesetzes zur Ausweitung der Mitwirkungsrechte des Bundestages in Angelegenheiten der EU der CDU/CSU-Fraktion, BT-Drs. 15/4716, S. 3, \$ 5 II 1: „Die Bundesregierung darf von der Stellungnahme des Deutschen Bundestages nur abweichen, wenn eine maßgeblich zu berücksichtigende Stellungnahme des Bundesrates oder wenn zwingende außen- und integrationspolitische Gründe dies erfordern." Aktuellere Bestrebungen der CSU gingen aus ihren Leitlinien (14-Punkte-Programm) hervor. Vgl. Christine Mellein, Die Rolle von Bundestag und Bundesrat in der Europäischen Union, in: EuR, 46. Jg. (2011) Beiheft 1, S. 13, S. 37, Fn. 110; Sebastian Gröning-von Thüna, Die neuen Begleitgesetze zum Vertrag von Lissabon aus Sicht des 
Im Folgenden soll geklärt werden, ob heute an der Forderung eines Weisungsrechts festzuhalten ist. Die Dringlichkeit dieser Forderung hängt wesentlich davon ab, wie weit der Istvom angestrebten Sollzustand abweicht. Inhaltlich geht es um die Bindung der Regierung an den Willen des Parlaments. Inwieweit der Bundestag bereits jetzt die Möglichkeit hat, die Regierung an seinen Willen zu binden, soll daher Ausgangspunkt der Überlegungen sein.

Die Regelung, die einem Weisungsrecht am nächsten kommt, ist $₫ 9$ IV EUZBBG. Danach muss die Bundesregierung einen Parlamentsvorbehalt im Europäischen Rat einlegen, wenn die Stellungnahme des Bundestages in einem wesentlichen Belang nicht durchsetzbar ist. Um einen konkreten Vergleich der dem Bundestag derzeit gesetzlich eingeräumten Befugnisse mit einem umfassenden Weisungsrecht in EU-Angelegenheiten zu ermöglichen, sollen im Folgenden Anwendungsbereich und Bindungswirkung der Stellungnahmen nach $\$ 9$ EUZBBG bestimmt werden.

\section{Anwendungsbereich von Stellungnahmen nach \$ 9 II und IV EUZBBG}

1.1. Unterscheidung in einfache und durch $\$ 9$ IV EUZBBG qualifizierte Stellungnahmen

Gemäß $\$ 9$ I EUZBBG gibt die Bundesregierung vor ihrer Mitwirkung an Vorhaben (definiert in $\$ 3$ EUZBBG) dem Bundestag Gelegenheit zur Stellungnahme. Gibt der Bundestag eine Stellungnahme ab, so „legt die Bundesregierung diese ihren Verhandlungen zugrunde“ (\$ 9 II 1 EUZBBG). Die Möglichkeit zur Stellungnahme muss die Regierung demnach geben, bevor sie in Verhandlungen tritt. Dass der Bundestag dazu inhaltlich imstande ist, wird durch die sich aus $\$ 4$ EUZBBG ergebende Pflicht der Bundesregierung zur umfassenden und frühestmöglichen Unterrichtung gewährleistet.

„Macht der Bundestag von der Gelegenheit zur Stellungnahme gemäß Artikel 23 Absatz 3 Satz 1 des Grundgesetzes Gebrauch, legt die Bundesregierung im Rat einen Parlamentsvorbehalt ein, wenn der Beschluss des Bundestages in einem seiner wesentlichen Belange nicht durchsetzbar ist" ( $\$ 9$ IV 1 EUZBBG). Dies betrifft die Fälle der Mitwirkung an Rechtsetzungsakten ${ }^{8}$ der EU durch die Bundesregierung. Vor der Abschlussentscheidung im Rat muss sich die Bundesregierung bemühen, Einvernehmen mit dem Bundestag herzustellen. Eine Abweichungsmöglichkeit aus wichtigen außen- oder integrationspolitischen Gründen steht der Bundesregierung gemäß $\$ 9$ IV 6 EUZBBG auch im Fall des eingelegten Parlamentsvorbehalts im Ergebnis zu. Im Umkehrschluss zu $\$ 9$ IV 6 EUZBBG gilt diese eingeschränkte Abweichungsmöglichkeit für beide Arten der Stellungnahme. Aufgrund der größeren Bedeutung erhalten die von $\$ 9$ IV EUZBBG erfassten Fälle die stärkere verfahrensrechtliche Absicherung. Damit wird sichergestellt, dass die Belange des Bundestages im Rat tatsächlich diskutiert werden.

Der Bundestag verfügt über einen ständigen Ausschuss für Angelegenheiten der Europäischen Union. ${ }^{9}$ Diesen kann er über Art. 45 S. 2 GG ermächtigen, für ihn die sich aus Art. 23 GG ergebenden Rechte, also auch das Stellungnahmerecht, gegenüber der Regierung wahrzunehmen.

Deutschen Bundestages - offene Fragen und neue Herausforderungen, in: integration, 33. Jg. (2010), H. 4, S. 312, S. 326.

8 Zum Begriff „Rechtsetzungsakt“ siehe Fußnote 13.

9 Vgl. Art. 45 Satz 1 GG sowie $\$ 54$ der Geschäftsordnung des Deutschen Bundstages. 


\subsection{Erfassung einer Teilmenge von Stellungnahmen in $\$ 9$ IV EUZBBG}

Die Unterscheidung von zwei Arten der Stellungnahme beruht auf der Annahme, dass $\$ 9$ IV EUZBBG nur eine Teilmenge von Stellungnahmen des $\$ 9$ II EUZBBG erfasst. Grund für diese Annahme ist, dass sich der weite Anwendungsbereich von Stellungnahmen nach \$\$ 9 I 1, 3 EUZBBG nicht mit dem des Art. 23 III 1 GG deckt. Aufgrund dieser, im Folgenden begründeten, Tatsache gewinnt der Verweis in $\$ 9$ IV 1 EUZBBG seine Bedeutung. Hätte der Gesetzgeber hingegen alle Vorhaben im Sinne von $\$ 3$ EUZBBG durch die Anforderungen des $\$ 9$ IV EUZBBG erfassen wollen, hätte der Verweis auf Art. 23 III 1 GG zwingend unterbleiben müssen. Beide Anwendungsbereiche sind so unterschiedlich groß, dass auch durch eine sehr extensive Auslegung des Art. 23 III 1 GG kein Gleichlauf herstellbar ist. Zwar ermöglicht eine extensive Auslegung die Erfassung von Maßnahmen, die das Vorfeld von Rechtsetzungsakten bilden. Jedoch bereiten nicht alle Vorhaben des $₫ 3$ EUZBBG Rechtsetzungsakte vor. Bei sehr engem Verständnis täten das nur „Vorschläge für Gesetzgebungsakte der EU“ (\$ 3 I Nr. 3 EUZBBG). Bei sehr weitem Verständnis könnte man eine Vielzahl von Aktionsplänen der Organe der EU sowie von Grün- und Weißbüchern der Kommission ( $\$ 3$ I Nr. 8, 9, 10 EUZBBG) als Rechtsetzungsakte vorbereitend qualifizieren. Bei Vorhaben nach $\$ 3$ I Nr. 4 bis 7 EUZBBG beispielsweise ist das jedoch grundsätzlich nicht möglich. An der bestehenden Divergenz vermag auch die Grundkonzeption des EUZBBG, Gesetz im Sinne von Art. 23 III 3 GG zu sein ${ }^{10}$, nichts zu ändern. Zudem steht diese Konzeption den Ausführungen nicht unbedingt entgegen. Die Funktion, Gesetz im Sinne von Art. 23 III 3 GG zu sein, erfüllt das EUZBBG. Es erschöpft sich aber nicht darin, sondern beansprucht darüber hinaus, die von Art. 23 II 1 GG geforderte Mitwirkung des Bundestages in Angelegenheiten der EU auszugestalten. ${ }^{11}$

Aber selbst wenn man (unzutreffenderweise) unterstellte, dass $\$ 9$ I 1 EUZBBG mit seinem weiten Vorhabenbegriff eine zulässige Konkretisierung des Art. 23 III 1 GG ist, würde dies am Bestehen der zwei Arten von Stellungnahmen nichts ändern. Man käme ebenfalls zum Ergebnis, dass der Verweis in \$9 IV EUZBBG kein überflüssiger, klarstellender Hinweis ist und Absatz 4 einen engeren (mit Art. 23 III 1 GG kongruenten) Anwendungsbereich als Absatz 2 hat. Der Verweis macht nämlich nur Sinn, wenn er sich auf den Kerngehalt des Art. 23 III 1 GG bezieht. Würde er auf Art. 23 III 1 GG in seiner durch Art. 23 III 3 GG i.V.m $₫ 3$ EUZBBG geprägten Form verweisen ${ }^{12}$, hätte er tatsächlich nur Klarstellungsfunktion. Er wäre aber viel zu umständlich, weil er über den Umweg des Art. 23 III 1 GG zurück auf den bereits in \$9 I 1 EUZBBG verwendeten Vorhabenbegriff des $\$ 3$ EUZBBG verweisen würde. Hätte der Gesetzgeber also tatsächlich gewollt, dass die Anforderungen von $\$ 9$ IV EUZBBG für alle Stellungnahmen gelten, hätte er einfach den Verweis auf Art. 23 III 1 GG unterlassen und $\$ 9$ IV 1 EUZBBG etwa wie folgt formuliert:

10 Siehe Fußnote zu Art. 23 III 3 GG, in: Carl Sartorius, Verfassungs- und Verwaltungsgesetze, München 2012.

11 Amtliche Begründung zu BT-Drs. 16/13925, S. 8.

12 Dies ist nur annehmbar, wenn man davon ausgeht, dass (1) das gesamte EUZBBG ein Gesetz im Sinne von Art. 23 III 3 GG ist und (2) zugleich über Art. 23 III 3 GG eine Definition der „Rechtsetzungsakte“ in Art. 23 III 1 GG ermöglicht wird. Gegen (2) spricht das Erfordernis der Geschlossenheit einer Verfassung. Sie muss aus sich heraus verständlich sein. Aus anderem Blickwinkel: Der Wortlaut der grundgesetzlichen Ermächtigung stellt die absolute Grenze der (einfach) gesetzlichen Gestaltungsmöglichkeit dar. 
Ist die Stellungnahme des Bundestages in einem seiner wesentlichen Belange nicht durchsetzbar, legt die Bundesregierung im Rat einen Parlamentsvorbehalt ein.

Auch die Gesetzessystematik spricht dafür, dass $\$ 9$ IV EUZBBG nicht für alle Stellungnahmen gilt. So unterscheidet auch $₫ 7$ EUZBBG zwischen Vorhaben im Sinne von $\$ 3$ EUZBBG und Vorschlägen für Gesetzgebungsakte ${ }^{13}$ und stellt für letztere eine erhöhte Anforderung an die Bundesregierung, nämlich eine Bewertung, die eine umfassende Folgenabschätzung enthält. Schließlich entspricht das Ergebnis der systematischen Auslegung auch der Intention des Gesetzgebers. Dies ergibt sich aus der amtlichen Begründung: $\$ 9$ „Absatz 1 verwendet den Vorhabenbegriff des $\$ 3$ und unterstreicht so den Anspruch des Bundestages, im Rahmen der Mitwirkung nach Artikel 23 Absatz 2 Satz 1 GG bei Bedarf zu allen Arten von Vorhaben Stellungnahmen abzugeben. Lediglich Absatz 4 bezieht sich ausschließlich auf Stellungnahmen des Bundestages zu Gesetzgebungsakten" ${ }^{14}$.

Es ist also festzuhalten, dass $\$ 9$ I 1, II 1 EUZBBG nicht Art. 23 III 1 GG ausgestaltet, sondern beide Arten der Stellungnahme nebeneinander bestehen. Genauso ist der den Art. 23 III 2 GG konkretisierende \$9 IV EUZBBG von dem „einfachen“ Stellungnahmerecht nach $\$ 9$ I 1, II 1 EUZBBG abgrenzbar. Dies ist möglich, weil $₫ 9$ IV EUZBBG aus sich heraus verständlich bleibt. Er stellt keinen Bezug zu $\$ 9$ I 1 EUZBBG und dessen Vorhabenbegriff dar und muss dies auch nicht, weil Art. 23 III 1 GG, auf den er verweist, eine vergleichbare Regelung enthält.

\section{Bindungswirkung von Stellungnahmen nach $\$ 9$ II und IV EUZBBG}

\subsection{Abstrakte Bindungswirkung}

Nach herrschender Ansicht in der Literatur haben Stellungnahmen als schlichte Parlamentsbeschlüsse keinerlei rechtliche Bindungswirkung. ${ }^{15}$ Auf eine lediglich politische Bindungs-

13 Art. 289 III AEUV definiert Gesetzgebungsakte. Unklar ist, ob der Begriff mit den „Rechtsetzungsakten“ in Art. 23 III 1 GG kongruent ist. Davon geht die Begründung zu BT-Drs. 16/13925, S. 8 aus, denn der Begriff Gesetzgebungsakte wird stellvertretend für Rechtsetzungsakte im Sinne von Art. 23 III 1 GG verwendet. Ebenso Wolfgang Fischer, Die Europäische Union im Grundgesetz - der neue Artikel 23, in: ZParl, 24. Jg. (1993), H. 1, S. 32 - 49, S. 32, S. 41. Von der Wortbedeutung ist „Rechtsetzung“" weiter als „Gesetzgebung“, vgl. Armin von Bogdandy, Stellungnahme zur gemeinsamen öffentlichen Anhörung der EU-Ausschüsse von Bundestag und Bundesrat, 26./27. August 2009, http://www.mpil.de/shared/data/pdf/stellungnahme.pdf (Abruf am 26. Mai 2012), S. 3, S. 20 - 22. Zu eventuellen Folgen der Bedeutungsdivergenz vgl. S. 399 f.

14 BT-Drs. 16/13925, S. 8; zum Begriff „Gesetzgebungsakt" siehe Fußnote 13.

15 Vgl. Rupert Scholz, in: Theodor Maunz / Günter Dürig, Grundgesetz. Kommentar, Bd. IV, München 2012, Art. 23, Rn. 159; Helge Sodan, in: ders. I Jan Ziekow, Grundkurs Öffentliches Recht, München 2010, S. 30; Sebastian Gröning-von Thüna, a.a.O. (Fn. 7), S. 326; Christoph Grabenwarter, Staatliches Unionsverfassungsrecht, in: Armin von Bogdandy / Jürgen Bast (Hrsg.) Europäisches Verfassungsrecht, Berlin 2009, S. 153, mit weiteren Nennungen in Fußnote 146; Christine Mellein, a.a.O. (Fn. 7), S. 37; Christian Calliess, Absicherung der parlamentarischen Integrationsund Budgetverantwortung auf europäischer und nationaler Ebene, in: Berliner Online-Beiträge zum Europarecht, Nr. 73 (2012), S. 1, S. 18 f. mit Verweis auf BT-Plenarprotokoll 16/52, S. 5067, auf S. 18 aber auch mit guten Argumenten für die hier vertretene Ansicht (andere Auffassung), Link auf http://www.portal-europarecht.de/ (Abruf am 26 Mai 2012); anderer Auffassung (wohl) Armin von Bogdandy, a.a.O. (Fn. 13), S. 24; Wolfgang Fischer, a.a.O. (Fn. 13), S. 41; Franz Möller 
wirkung eines einfachen Parlamentsbeschlusses abzustellen, greift im europarechtlichen Kontext jedoch zu kurz.

Dies verdeutlicht ein Vergleich mit $\$ \$ 5$ I, 6 I, 9 I und 10 IntVG. Es handelt sich dabei um Parlamentsbeschlüsse mit Rechtsverbindlichkeit. $\$ 5$ I und 6 I IntVG betreffen die Zustimmung der Bundesregierung im Europäischen Rat zu besonderen Brückenklauseln. Zustimmen oder enthalten darf sich der jeweilige Regierungsvertreter im Rat nur, wenn der Bundestag hierzu zuvor einen Beschluss gefasst hat. Fehlt dieser, muss der deutsche Vertreter im Europäischen Rat jeden der dort genannten Beschlussvorschläge ablehnen. Damit stellt dieser Parlamentsbeschluss ein zwingendes Mitwirkungsrecht dar, ohne den der jeweilige Ratsbeschluss nicht zustande kommt. In den in $\$ 9$ I IntVG geregelten Fällen (Notbremsemechanismus) muss der durch Parlamentsbeschluss angewiesene Regierungsvertreter beantragen, den Rat zu befassen. Die rechtliche Bindungswirkung wird hier durch die Worte „muss" und „durch Beschluss angewiesen hat" zum Ausdruck gebracht.

Auch dem ablehnenden Parlamentsbeschluss nach $\$ 10$ I, III IntVG kommt Rechtsverbindlichkeit zu. Rechtsfolge ist, dass der angestrebte Ratsbeschluss nicht erlassen wird. ${ }^{16}$ Eine Abstimmung im Europäischen Rat findet also nicht statt. Damit löst der Parlamentsbeschluss das Scheitern der Initiative unmittelbar aus, sobald der Bundestag die zuständigen EU-Organe gemäß $₫ 10$ II IntVG davon in Kenntnis setzt. Ohne diesen ablehnenden Beschluss könnte der Bundestag die Initiative erst zu einem späteren Zeitpunkt und nur mittelbar über die sich aus $\$ 4$ IntVG ergebende Ablehnungsverpflichtung des deutschen Vertreters im Europäischen Rat scheitern lassen.

Die Formulierung in Art. 23 III 1 GG „Die Bundesregierung berücksichtigt Stellungnahmen des Bundestages bei den Verhandlungen " lässt Raum für eine rechtliche Verbindlichkeit. Die aktive Formulierung „berücksichtigt“ bedeutet im GG „hat zu berücksichtigen“. Um eine Ermessensvorschrift handelt es sich nicht, da eine solche im GG durch „soll“ oder „kann“ hervorgehoben wird. Mangels Ermessens ist also eine gewisse tatsächliche Bindung der Bundesregierung vom GG vorgesehen. Die bestehende rechtliche Verpflichtung der Bundesregierung, die Stellungnahmen in ihre Entscheidung ${ }^{17}$, zumindest aber in ihre Entscheidungsfindung ${ }^{18}$ einzubeziehen, wird in der Literatur nicht bestritten. Dies relativiert jedoch bereits die Behauptung der fehlenden Rechtsverbindlichkeit. Die Position der herrschenden Lehre ist folglich dahingehend zu präzisieren, dass keine rechtliche Bindung der Regierung an den Inhalt der Stellungnahme bei ihrer Entscheidung im Rat besteht. Ihr kann darin gefolgt werden, dass der Wortsinn des Art. 23 III 1 GG diesbezüglich keine Verbindlichkeit festschreibt. Allerdings engt die Formulierung „berücksichtigt ... bei den Verhandlungen“ den Bundestag auch nicht dahingehend ein, bei der Ausgestaltung von Art. 23 III 1 GG seinen Stellungnahmen nur politische Verbindlichkeit zu geben. ${ }^{19}$

/ Martin Limpert, Informations- und Mitwirkungsrechte des Bundestages in Angelegenheiten der EU, in: ZParl, 24. Jg. (1993), H. 1, S. 21 - 32, S. 21, S. 29, die ausdrücklich eine „rechtliche Verpflichtung" zur Einbeziehung der Stellungnahme in die Entscheidung beziehungsweise Entscheidungsfindung anerkennen.

16 Vgl. Art. 48 III UA 3, Satz 2 EUV; Art. 81 III UA 3, Satz 2 AEUV.

17 Vgl. Wolfgang Fischer, a.a.O. (Fn. 13), S. 41 f.; BT-Drs. 12/3896, S. 20.

18 Vgl. zum Beispiel Rupert Scholz, a.a.O. (Fn. 15), Rn. 159; Helge Sodan, a.a.O. (Fn. 15), S. 30; Franz Möller / Martin Limpert, a.a.O. (Fn. 15), S. 27 ff.; Christine Mellein, a.a.O. (Fn. 7), S. 37.

19 In dem durch den Wortlaut beziehungsweise den Wortsinn begrenzten Rahmen, ist eine Abweichung von der im allgemeinen Sprachgebrauch vorherrschenden Bedeutung nach „oben“ (in Rich- 
Und dies hat er auch nicht getan. Vielmehr regelt $\$ 9$ IV EUZBBG ausdrücklich, wie weit die (rechtliche) Bindungswirkung geht. Eine Abweichung, und zwar in der Entscheidung, ist nur aus wichtigen außen- und integrationspolitischen Gründen zulässig. Der Wortlaut „abweichende Entscheidungen“ des $\$ 9$ IV 6 EUZBBG verdeutlicht, dass es hier nicht um den Entscheidungsfindungsprozess, sondern um die Entscheidung selbst geht. Der Umkehrschluss legt nahe, dass es in den übrigen Fällen des $\$ 9$ IV EUZBBG eine Bindung in der Entscheidung gibt. Diese Abweichungsmöglichkeit gilt für alle Stellungnahmen des $\$ 9$ EUZBBG, erlangt jedoch in den Fällen der einfachen Stellungnahme kaum praktische Bedeutung. ${ }^{20}$

Damit ist die Stellungnahme im europarechtlichen Kontext zu einem Instrumentarium eigener Art geworden, deren Bindungswirkung nur durch die gesetzliche Ausgestaltung selbst, nicht aber abstrakt mit Hinweis auf den Beschlusscharakter bestimmbar ist. ${ }^{21}$

\subsection{Konkrete Bindungswirkung von Stellungnahmen nach $\$ 9$ II und IV EUZBBG}

Wozu genau die Bundesregierung verpflichtet ist, wenn kein Abweichungsgrund vorliegt, kann demnach nur $\$ 9$ EUZBBG selbst beantworten.

An einer am konkreten Wortlaut orientierten Antwort könnte man sich indes aufgrund der Wortlautdivergenz von Grundgesetz und EUZBBG gehindert sehen. In Art. 23 III 2 GG heißt es, dass die Bundesregierung die Stellungnahmen des Bundestages „,bei den Verhandlungen berücksichtigt", während $\$ 9$ Abs. 2 EUZBBG davon spricht, dass die Bundesregierung die Stellungnahme „ihren Verhandlungen zu Grunde legt“. „Zugrunde legen“ bezieht sich anders als „berücksichtigen“ nur auf den Anfang des Willensbildungsprozesses. ${ }^{22}$ Dieser Bedeutungsunterschied führt zu einer unterschiedlichen zeitlichen Anknüpfung für eine Bindungswirkung. Der Zeitraum, in dem die Stellungnahme von der Bundesregierung zu beachten ist, ist nach $\$ 9$ II EUZBBG kürzer. Der durch das Einstimmigkeitserfordernis im Rat ausgeübte Druck, einen Konsens zu finden, zwingt die Ratsmitglieder dazu, Abstriche von ihren Positionen zu machen und wenn diese überhaupt nicht konsensfähig sind, irgendwann ganz aufzugeben. Dies kann nach fortschreitenden Verhandlungen dazu führen, dass die ursprüngliche, durch die Stellungnahme festgelegte Position der Regierung keine Beachtung bei der abschließenden Entscheidung im Rat findet. Die rechtliche Verpflichtung

tung Verbindlichkeit) oder „unten“ (nur Kenntnisnahme) möglich. Eine Abweichung nach „unten" ist hier allerdings durch den Regelungszusammenhang gesperrt. Es geht im Rahmen des Art. 23 GG um eine Kompensation des mit der Übertragung von Hoheitsrechten einhergehenden Machtverlustes des Bundestages an sich und im Verhältnis zur Regierung. Die EU-Gesetzgebung beeinflussen, kann der Bundestag ausschließlich durch Einwirkung über den Regierungsvertreter auf den Rat. Die effektive Nutzung dieser Einwirkungsmöglichkeit wäre die bestmögliche Kompensation und damit im Sinne des Regelungskomplexes. Effektiv ist die Stellungnahme nur, wenn sich die Regierung an sie halten muss. Folglich ist eine Abweichung nach „oben“ im Rahmen der Wortlautgrenze zulässig.

20 Hier ist ohnehin eine Abweichung möglich (vgl. nachfolgend).

21 Diese Erkenntnis ist nicht neu. Franz Möller / Martin Limpert, a.a.O. (Fn. 15), S. 28, sprechen von einem „verfassungsrechtlichen Novum“ sowie einer „Fortentwicklung des Instituts der schlichten Parlamentsbeschlüsse“.

22 Auf diese Auslegung einigte sich der Bundestags-Sonderausschuss „Europäische Union (Vertrag von Maastricht)“, vgl. BT-Drs. 12/3896, S. 19. 
der Regierung, die Stellungnahme zu beachten, besteht folglich nicht bis zur Schlussabstimmung im Rat fort und entfaltet dort keinerlei Bindung mehr. Art. 23 III GG beinhaltet diese zeitliche Einschränkung nicht. Die Formulierung „berücksichtigt“ ist weiter. Stellungnahmen sind danach beim gesamten Willensbildungsprozess von der Regierung zu beachten. ${ }^{23}$ Eine rechtliche Bindung besteht also bis zur Schlussabstimmung fort. Hiermit ist zwar nicht eine inhaltliche Bindung an die Stellungnahme gemeint. Sie ist aber als wesentlicher Aspekt in den Abwägungsprozess, der unmittelbar die Abschlussentscheidung herbeiführt, einzustellen. ${ }^{24}$ Man kann das auch als Rechtspflicht, sich mit der Stellungnahme bei der Abschlussentscheidung auseinanderzusetzen, bezeichnen. ${ }^{25}$ Bei $\$ 9$ II EUZBBG besteht diese Auseinandersetzungspflicht hingegen nur bei der Festlegung der Verhandlungsposition. Um eine zulässige Konkretisierung durch $\$ 9$ II EUZBBG kann es sich bei der Bedeutungsdivergenz nicht handeln, weil Art. 23 GG insofern die Mindestanforderungen der parlamentarischen Kontrolle statuiert, die zwar über-, nicht aber unterschritten werden dürfen. ${ }^{26}$

Die bestehende inhaltliche Differenz könnte durch verfassungskonforme, das heißt an Art. 23 III 2 GG orientierte Auslegung des $\$ 9$ II EUZBBG aufzulösen sein. ${ }^{27}$ Diese Lesart führt dazu, dass jegliche Stellungnahmen eine rechtliche Bindungswirkung dahingehend entfalten, dass sie bei der Abschlussentscheidung im Rat als wesentlicher Aspekt Beachtung finden müssen. Erfasst wären alle Vorhaben des $\$ 3$ EUZBBG sowie vergleichbare, da $\$ 3$ EUZBBG nicht abschließend ist. Im Zusammenspiel mit der durch $₫ 9$ IV 6 EUZBBG 28 stark eingeschränkten Abweichungsmöglichkeit liefe dies auf ein sehr umfangreiches Berücksichtigungsgebot in Angelegenheiten der EU hinaus. Jenes, so wird befürchtet, könnte an die Grenzen des nach Art. 23 II, III ${ }^{29}$ GG Zulässigen führen und die europapolitische Handlungsfähigkeit der Regierung beeinträchtigen. Letzterem möchte Rupert Scholz, mit Hinweis auf die nach dem Grundgesetz erforderliche Wahrung des Kernbereichs exekutiver Eigenverantwortung, durch eine im Einzelfall einschränkende Auslegung des $₫ 9$ II EUZBBG begegnen. ${ }^{30}$

Derartige Komplikationen sind jedoch entbehrlich. Unter Beachtung der oben ausgeführten Unterscheidung der zwei Arten von Stellungnahmen handelt es sich hier genau genommen gar nicht um verfassungskonforme Auslegung. Art. 23 III 2 GG erfordert diese Auslegung ebenso wenig, wie er eine rechtliche Bindung der Regierung in allen Fällen des $\$ 3$ EUZBBG verlangt. Er verlangt eine Bindung nur in den Fällen des $\$ 9$ IV EUZBBG. Außerhalb des Anwendungsbereiches des Art. 23 III 2 GG ist es daher unproblematisch, dass letztlich keine rechtliche Bindung an die Stellungnahme des Bundestages besteht, weil die Regierung diese ihren Verhandlungen „nur“ zugrunde legt. So ist zugleich die Hand-

23 So auch das Ergebnis des Bundestags-Sonderausschusses, vgl. BT-Drs. 12/3896, S. 19.

24 Der Wortlaut des $\$ 9$ IV EUZBBG blieb dabei unberücksichtigt, weil es hier nur um die Frage geht, inwieweit der Formulierung „berücksichtigt ... bei den Verhandlungen“ gemäß Art. 23 III 2 GG Bindungswirkung zukommt. Zur rechtlichen Verbindlichkeit in der Entscheidung kommt es erst über $\$ 9$ IV EUZBBG.

25 Vgl. BT-Drs. 12/3896; Wolfgang Fischer, a.a.O. (Fn. 13), S. 41 f.

26 Dies ergibt sich aus dem Regelungszusammenhang mit Art. 23 GG im Übrigen, vgl. Fußnote 19.

27 Rupert Scholz, a.a.O. (Fn. 15), Rn. 158.

28 Genau genommen ergibt erst der Umkehrschluss aus der Norm, dass diese Abweichungsmöglichkeit für alle Stellungnahmen gilt.

29 Vgl. Armin von Bogdandy, a.a.O. (Fn. 13), S. 24.

30 Vgl. Rupert Scholz, a.a.O. (Fn. 15), Rn. 158. 
lungsfähigkeit der Regierung sichergestellt. Insoweit bedarf es der von Rupert Scholz vorgeschlagenen korrigierenden Auslegung nicht.

Wenn überhaupt, hat eine verfassungskonforme Auslegung des $\$ 9$ II EUZBBG nur im Anwendungsbereich von Stellungnahmen nach $\$ 9$ IV EUZBBG seine Berechtigung. Aber auch hier scheint sie entbehrlich zu sein ${ }^{31}$, denn $\$ 9$ IV EUZBBG erweitert die zeitliche Bindungswirkung der Stellungnahme gegenüber $\$ 9$ II EUZBBG und stellt so einen Gleichlauf mit Art. 23 III 2 GG her. Die Bundesregierung muss nämlich einen Parlamentsvorbehalt einlegen, wenn die Stellungnahme nicht durchsetzbar ist. Damit ist diese im gesamten Willensbildungsprozess, also bis zur Schlussabstimmung im Rat, von der Regierung zu beachten. Dies entspricht exakt der gängigen Auslegung von „berücksichtigt bei den Verhandlungen“ gemäß Art. 23 III 2 GG. Die aufgrund der Bedeutungsdivergenz von „berücksichtigen“ und „zugrunde legen“ unterschiedlichen zeitlichen Bindungswirkungen werden also durch das Berücksichtigungsgebot, das von dem einzulegenden Parlamentsvorbehalt ausgeht, zunächst einmal eingeebnet.

Dabei lässt es $\$ 9$ IV EUZBBG jedoch nicht bewenden. Er erweitert die Bindungswirkung auch qualitativ gegenüber Art. 23 III 2 GG. Soweit keine „wichtigen außen- oder integrationspolitischen Gründe“ eine abweichende Entscheidung rechtfertigen, ist diese Stellungnahme für die Regierung rechtlich bindend. Das Besondere ist, dass hier im Regelfall tatsächlich eine Bindung an den wesentlichen Inhalt der Stellungnahme besteht. Dies wird dadurch erreicht, dass der Parlamentsvorbehalt eingelegt werden muss, wenn die Stellungnahme in einem ihrer „wesentlichen Belange nicht durchsetzbar“ ist. Die Regierung muss also versuchen, die Stellungnahme im Rat durchzusetzen; ihre Position muss somit im Wesentlichen der Stellungnahme entsprechen. Dass sie dieser rechtlichen Verpflichtung nachkommt, ist prozessual über den Parlamentsvorbehalt abgesichert, zu dessen Einlegung die Regierung rechtlich verpflichtet ist, wenn ihr die Durchsetzung der Stellungnahme nicht gelingt. Der Wortlaut „abweichende Entscheidungen“ des $\$ 9$ IV 6 EUZBBG verdeutlicht, dass es hier nicht um den Entscheidungsfindungsprozess, sondern um die Entscheidung selbst geht. Die systematische Stellung hinter Satz 4 und auch die inhaltliche Bezugnahme auf diesen stellen klar, dass hier nur die Abschlussentscheidung im Rat gemeint sein kann. Aus dem Umkehrschluss zu $\$ 9$ IV 6 EUZBBG ergibt sich, dass es in den übrigen Fällen des $\$ 9$ IV EUZBBG, also in der Regel, eine Bindung in der Entscheidung gibt. Daran ändert auch die erforderliche Bemühung der Regierung um Einvernehmen nichts, denn erzwingen kann sie das Einvernehmen des Bundestages nicht. Gelingt es nicht, besteht die Bindung auch nach dieser Bemühung bis zur Abschlussentscheidung in allen Fällen fort, in denen

31 Verfassungskonformer Auslegung bedürfte es nur, wenn nach dem erfolglosen Bemühen der Regierung um Einvernehmen noch so umfangreiche Verhandlungen im Rat stattfinden, dass diese bei bloßem „zu Grunde legen“ der (erneuerten) Stellungnahme deren Unbeachtlichkeit in der Abschlussentscheidung rechtfertigen. Bei fehlender Pflicht zur Einbeziehung in die Abschlussentscheidung bliebe die eingeschränkte Abweichungsmöglichkeit nämlich wirkungslos. Das dürfte \$ 9 IV 4 EUZBBG aber verhindern, da das Einvernehmen „vor“ der „abschließenden“ Entscheidung hergestellt werden soll und somit das Zeitfenster für das geschilderte Szenario zu klein sein dürfte. Befürwortet man hingegen im Sinne der Einheitlichkeit, aufgrund einer im Ausnahmefall notwendigen verfassungskonformen Auslegung, dieselbe Auslegung für alle Fälle des $\$ 9$ IV EUZBBG, hat dies den Nachteil, dass „zugrunde legen“ in \$9 II EUZBBG zwei unterschiedliche Bedeutungen erhält. 
kein Abweichungsgrund gegeben ist. ${ }^{32}$ Für das gefundene Ergebnis sprechen auch teleologische Erwägungen. So lässt sich anführen, dass $\$ 9$ IV 6 EUZBBG schlichtweg überflüssig wäre, wenn sich auch im Falle fehlenden Einvernehmens die Regierungsposition durchsetzen würde. ${ }^{33}$ Auch der von der Regierung einzulegende Parlamentsvorbehalt könnte dann seine Funktion „im Ernstfall“ kaum noch erfüllen. Bei einer verfestigten Regierungsposition bestünde so die Gefahr, dass die Einlegung des Parlamentsvorbehalts und das ,Bemühen um Einvernehmen als rein „formalbürokratische Amtshandlungen“ abgearbeitet werden.

Zwar erfordert das Berücksichtigungsgebot des Art. 23 III 2 GG kein derart eingeschränktes Abweichungsrecht, so dass diesem sicherlich auch mit einer großzügigeren Abweichungsmöglichkeit genüge getan wäre. Der Gesetzgeber kann jedoch (per Gesetz nach Art. 23 III 3 GG) in gewissem Umfang über die durch Art. 23 III 1, 2 GG fixierten Mindestanforderungen an die parlamentarische Kontrolle der Regierung hinausgehen ${ }^{34}$, dies insbesondere, weil die Formulierung „,bei den Verhandlungen berücksichtigt“ der einfachgesetzlichen Ausgestaltung aufgrund ihrer Abstraktheit sehr zugänglich ist.

Im Hinblick auf $\$ 9$ IV EUZBBG gibt es noch eine weitere Erklärungsmöglichkeit. Dabei geht man nicht von einer qualifizierten Teilmenge von Stellungnahmen im Sinne von $\$ 9$ I, II EUZBBG aus, sondern von einem aliud, also einem davon losgelösten, eigenständigen, mit Art. 23 III GG einheitlichen Stellungnahmerecht.

Versteht man $\$ 9$ IV EUZBBG als aliud, bedarf es des Rückgriffs auf $₫ 9$ II EUZBBG und dessen Auslegung nicht, weil dann unmittelbar das „Berücksichtigen“ des Art. 23 III 2 GG gilt. Dem steht nicht entgegen, dass $₫ 9$ IV EUZBBG nur zu Art. 23 III 1 GG einen Bezug herstellt. Anliegen von $\$ 9$ IV EUZBBG ist die Konkretisierung von Art. 23 III 2 GG, denn er ist ausgestaltendes Gesetz im Sinne des Art. 23 III 3 GG. Ein Hinweis auf Art. 23 III 2 GG wäre daher funktionslos. Wenn also $\$ 9$ IV EUZBBG nur eine Konkretisierung im Sinne von Art. 23 III 3 GG des Stellungnahmerechts aus Art. 23 III 1, 2 GG ist, gilt Art. 23 III 2 GG direkt für diese Art der Stellungnahme. Ein Rückgriff auf $\$ 9$ II EUZBBG ist nach diesem Verständnis weder erforderlich noch zulässig.

Vorteil dieser Erklärungsmöglichkeit ist seine Einfachheit. Fraglich ist jedoch, ob die Annahme eines losgelösten Stellungnahmerechts zutreffend ist. Der geäußerte Wille des Gesetzgebers („lediglich Absatz 4 bezieht sich ausschließlich auf Stellungnahmen des Bundestages zu Gesetzgebungsakten “35) steht dem jedenfalls nicht entgegen. Probleme könnten bei einer strikten Trennung in der Praxis auftreten. So könnten die $\$ \$ 4$ bis 7 EUZBBG, die an den Vorhabenbegriff des $\$ 3$ EUZBBG anknüpfen, für das Stellungnahmerecht aus Art. 23 III GG, $\$ 9$ IV EUZBBG nicht anwendbar sein. Das ist soweit unschädlich, als über $\$ 3$ I Nr. 3 i.V.m. $\$ \$ 4$ bis 7 EUZBBG bereits eine Versorgung des Bundestages mit Informationen gewährleistet ist. Problematisch könnte indes die bestehende Divergenz zwischen den Begriffen „Rechtsetzungsakte der EU“ (Art. 23 III 1 GG) und „Gesetzgebungsakte“ (\$ 3 I Nr. 3. EUZBBG) sein. ${ }^{36}$ So könnte es möglich sein, dass einige Rechtsetzungsakte,

$32 \mathrm{Zu}$ beachten ist die eventuelle Einschränkung in Fußnote 31.

33 Vgl. Christian Calliess, a.a.O. (Fn. 15), S. 18.

34 Vgl. Fußnote 19.

35 BT-Drs. 16/13925, S. 8. „Gesetzgebungsakte“ meint „Rechtsetzungsakte der EU“ im Sinne von Art. 23 III 1 GG.

36 Vgl. Armin von Bogdandy, a.a.O. (Fn. 13), S. 3, S. 20 ff. 
die nicht Gesetzgebungsakte im Sinne von $₫ 3$ Nr. 3 EUZBBG sind, von $₫ 3$ EUZBBG überhaupt nicht erfasst werden und damit die an $\$ 3$ EUZBBG anknüpfenden Unterrichtungspflichten nicht für alle Fälle des $\$ 9$ IV EUZBBG gelten würden. In diesem Fall hilft jedoch die Tatsache, dass $\$ 3$ EUZBBG nicht abschließend ist. Dass es sich bei $\$ 3$ I EUZBBG um Regelbeispiele handelt, wird durch das Wort „insbesondere“ zum Ausdruck gebracht. Aufgrund der Vergleichbarkeit der geregelten Sachverhalte zu den nicht geregelten und der Wichtigkeit, die durch eine Regelung in der Verfassung zum Ausdruck gebracht wird, ist davon auszugehen, dass im Ergebnis doch alle Fälle von Art. 23 III GG von $\$ 3$ EUZBBG erfasst werden.

Gegen das Bestehen eines völlig eigenständigen Stellungnahmerechts spricht jedoch die Systematik. Hätte der Gesetzgeber ein solches für die Fälle des Art. 23 III GG gewollt, hätte er beide Stellungnahmerechte weder in denselben Paragraphen geschrieben noch mit einer gemeinsamen amtlichen Überschrift versehen. Zudem beinhaltet $₫ 9$ IV EUZBBG mit seinem Abweichungsrecht eine Voraussetzung, die auch für die übrigen Stellungnahmen gilt. Gerade die Formulierung „bleibt unberührt“ verdeutlicht, dass trotz der erhöhten Anforderungen noch von einem "gemeinsamen Nenner“, also einem einheitlichen Stellungnahmerecht ausgegangen wird. Auch wäre andernfalls die Handhabbarkeit des $₫ 9$ EUZBBG kompliziert, weil nicht klar wäre, welche prozessualen Anforderungen, etwa von $\$ 9$ I 2, II 2, III, V EUZBBG, für beide Arten der Stellungnahme gelten. Im Ergebnis ist daher nicht von einem völlig selbständigen Stellungnahmerecht in $\$ 9$ IV EUZBBG auszugehen und der oben beschriebene Ansatz, der von einer qualifizierten Stellungnahme bei einheitlichem Stellungnahmerecht ausgeht, vorzugswürdig.

\subsection{Europäischer Vergleich}

Durch die sehr eingeschränkte Abweichungsmöglichkeit bindet $\$ 9$ IV EUZBBG die Regierung in einem starken, nach Art. 23 III 1, 2 GG noch zulässigen Maße an den Willen des Parlaments. ${ }^{37}$ Über eine vergleichbar strenge (gesetzliche) Bindung der Verhandlungsführung der Regierung verfügen sonst nur wenige Mitgliedstaaten. ${ }^{38}$ In der Verfassung Österreichs ${ }^{39}$ findet sich eine ähnlich stark beschränkte Abweichungsmöglichkeit der Regierung wieder. In Dänemark benötigt der Regierungsvertreter stets eine Parlamentsmehrheit für seine Position, um diese im Rat vertreten zu können. Ist dies nicht der Fall, muss er eine mehrheitsfähige Position erarbeiten. ${ }^{40}$ In Großbritannien steht die Zustimmung des Rats-

37 Vgl. ebenda S. 24. Anderer Auffassung Sebastian Gröning-von Thüna, a.a.O. (Fn. 7), S. 326: „Binden kann der Bundestag die Bundesregierung ... nicht." Ebenso Christine Mellein, a.a.O. (Fn. 7), S. 37; entspricht der herrschenden Lehre (Fußnote 15 mit weiteren Nennungen).

38 Dänemark, Estland, Litauen, Slowakei, Slowenien und Schweden praktizieren eine parlamentarische „Mandatierung“, von der ihre Regierungen kaum abweichen; vgl. COSAC, Third Be-annual Report: Developments in EU Procedures and Practices Relevant to Parliamentary Scrutiny, Luxemburg 2005, S. 12 f., http://www.cosac.eu/en/info/scrutiny/reports.pdf (Abruf am 26. Mai 2012).

39 Vgl. Art. 23 e Abs. 2 bis 4 des österreichischen Bundes-Verfassungsgesetz (B-VG): ,aus zwingenden außen- und integrationspolitischen Gründen".

40 Vgl. Arthur Benz / Jörg Broschek, Beitrag für die Friedrich Ebert Stiftung im März 2010: Nationale Parlamente in der europäischen Politik - Funktionen, Probleme und Lösungen, S. 15, http// library.fes.de/pdf-files/id/ipa/07084.pdf (Abruf am 23. Mai 2012); Maja Møller Sousa, Learning 
vertreters unter einem Parlamentsvorbehalt, der dem Unterhaus oder dem Kontrollausschuss eine Vorabprüfung ermöglicht. Ohne dieses Prüfungsergebnis ist eine Zustimmung im Rat nur unter sehr engen Voraussetzungen möglich. ${ }^{41}$ Die Mehrzahl der Mitgliedstaaten verlangt hingegen nur eine Berücksichtigung der Stellungnahme und eine Darlegung der Gründe im Fall des Abweichens. ${ }^{42}$

Zwar darf die Bedeutung des Stellungnahmerechts in der (deutschen) Praxis nicht überschätzt werden. Die Bundestagsmehrheit hat kein Interesse an einer öffentlichen Kritik ihrer Regierung und wird vom Stellungnahmerecht zurückhaltend Gebrauch machen. ${ }^{43}$ Auf europäischer Ebene wird jedoch auch die theoretische Einflussnahmemöglichkeit der nationalen Parlamente (über ihre Regierungen) auf die Ratsbeschlüsse genau wahrgenommen. ${ }^{44}$ Eine stark ausgeprägte parlamentarische Einflussnahmemöglichkeit wird von anderen Mitgliedstaaten eher als Indiz für eine gewisse Skepsis gegenüber (Entscheidungsprozessen in) der EU gewertet. ${ }^{45}$ Eine Ursache dafür könnte sein, dass nationale Parlamente, aufgrund der regionalen Verbundenheit ihrer Abgeordneten, nationale Belange oft stärker gewichten als Regierungsvertreter. Auch stellt ein „starkes“ Parlament, neben dem Ratsvertreter, einen weiteren Akteur dar, der Ratsentscheidungen gegebenenfalls zugunsten seines Mitgliedstaates beeinflussen kann.

Andererseits haben Stellungnahmen eines Parlaments, unabhängig von ihrer Bindungswirkung gegenüber der Regierung, einen Einfluss auf andere Mitgliedstaaten. Aufgrund der guten informatorischen Vernetzung der nationalen Parlamente werden diese Stellungnahmen den zuständigen Ausschüssen umgehend zugeleitet und werden so zum Gegenstand des Entscheidungsfindungsprozesses anderer nationaler Parlamente. Dies stellt eine konstruktive Möglichkeit für den Bundestag dar, früh auf Entscheidungsprozesse in anderen Mitgliedstaaten Einfluss zu nehmen. Die Wirkung sollte nicht unterschätzt werden.

\section{Folgenbetrachtung eines Weisungsrechts}

Die Einräumung eines Weisungsrechts gegenüber der Bundesregierung würde von anderen Mitgliedstaaten wohl als Schaffung einer weiteren Möglichkeit, Entscheidungen in der EU notfalls verhindern zu können, verstanden werden. Deutschland könnte damit Gefahr laufen, seine bisherige Rolle als Integrationsmotor der EU unfreiwillig gegen eine Vorbildrolle

in Denmark? The Case of Danish Parliamentary Control over European Union Policy, in: Scandinavian Political Studies, 31. Jg. (2008), H. 4, S. 428 - 447, S. 432 f.

41 Vgl. Arthur Benz / Jörg Broschek, a.a.O. (Fn. 40), S. 16; Department of the Clerk of the House, The European Scrutiny System in the House of Commons. A Short Guide for Members of Parliament By the staff of the European Scrutiny Committee, London 2009, http://www.parliament. uk/documents/upload/theeuroscrutinysysteminthehoc.pdf/ (Abruf am 26. Mai 2012).

42 Vgl. Armin von Bogdandy, a.a.O. (Fn. 13), S. 24; Christoph Grabenwarter, a.a.O. (Fn. 15), S. 149 ff.

43 Vgl. Armin von Bogdandy, a.a.O. (Fn. 13), S. 24.

44 Zum Beispiel durch die Arbeit der COSAC (Conférence des Organes Spécialisés dans les Affaires Communautaires, deutsch: Konferenz der Europa-Ausschüsse der nationalen Parlamente). Informationen zu COSAC unter http://www.bundestag.de/service/glossar/C/cosac.html; http://www. cosac.eu (Abruf jeweils am 26. Mai 2012).

45 Die Ausgestaltung parlamentarischer Regierungskontrolle sollte, auch weil Skepsis nicht per se als negativ anzusehen ist, davon nicht abhängen. 
für Europaskeptiker einzutauschen. ${ }^{46}$ Damit allein sollte der Verzicht auf ein Weisungsrecht aber nicht begründet werden.

Für den Bundestag wäre ein Weisungsrecht sicherlich angenehm. Neben dem Machtzuwachs, der mit der im Einzelfall erforderlichen Ausübung des Weisungsrechts einhergeht, könnte allein das Bestehen dieser Handlungsoption schon disziplinierend auf die Regierung wirken. Man sollte jedoch die Konsequenz der Möglichkeit bedenken, dass die anderen Mitgliedstaaten dem deutschen Beispiel folgen. Wenn andere Mitgliedstaaten die deutsche Positionierung ermitteln, findet letztlich die gesetzliche Regelung maßgebliche Beachtung. Der Hinweis oder die Tatsache, dass der Deutsche Bundestag zurückhaltend von diesem Recht Gebrauch machen wird ${ }^{47}$, vermag andere Mitgliedstaaten nach dessen Einführung nicht abzuhalten, davon intensiven Gebrauch zu machen. Der Wirkung, die eine intensive Nutzung des Weisungsrechts durch die nationalen Parlamente zeitigt, sollte man sich daher bewusst sein. Die Konsensbildung im Rat wäre erheblich erschwert. ${ }^{48} \mathrm{Hat}$ etwa der deutsche Ratsvertreter einen für Europa sinnvollen Vorschlag und vermag er die anderen Regierungsvertreter davon zu überzeugen, wäre die Mühe bei entgegenstehender Weisung nationaler Parlamente umsonst. Folge wäre, dass der deutsche Ratsvertreter zusätzlich vor den nationalen Parlamenten seine Position vertreten müsste, um einen positiven Ratsbeschluss zu erzielen. Die Entscheidungsfindung wäre extrem erschwert, weil diejenigen, die es zu überzeugen gilt (die nationalen Parlamente), bei den Ratssitzungen nicht anwesend sind. Eine zügige Konsensbildung wäre unmöglich, weil erstens kein Regierungsvertreter weiß, ob sich sein Parlament auf einen neuen Konsensvorschlag einlässt, und zweitens die Willensbildung in den Parlamenten naturgemäß eine gewisse Zeit beansprucht. Der Entscheidungsfindungsprozess im Rat könnte durch Weisungen extrem verlängert und schließlich völlig ineffizient werden.

Dänemark, das lange Zeit als Modell für ein funktionierendes Weisungsrecht galt, bekommt zunehmend Probleme damit, insbesondere aufgrund der Einführung von Mehrheitsentscheidungen im Rat ${ }^{49}$, dies trotz der Tatsache, dass dem Regierungsvertreter in der Praxis regelmäßig ein gewisser Verhandlungsspielraum eingeräumt wird. ${ }^{50}$ Dies stützt die Annahme, dass selbst für kleinere Mitgliedstaaten ein alleiniges Setzen auf eine Art „Vetorecht“ nicht mehr zeitgemäß ist. Dem Erfordernis der aktiven Beteiligung an der europäischen Willensbildung können sich die nationalen Parlamente durch ein Weisungsrecht jedenfalls nicht mehr entziehen.

Aufgrund seiner Größe und Einflussmöglichkeiten kann und sollte Deutschland seine Interessen eher durch aktive (Mit-)Gestaltung der EU durchsetzen als durch Blockierung von Entscheidungen. Nur durch konstruktive Europapolitik können neue politische Projekte, wie etwa das Vorantreiben der Nutzung erneuerbarer Energien, europaweit umgesetzt werden. Wie sehr diese Politik im nationalen Interesse sein kann, zeigt sich am Beispiel umweltbelastender Emissionen. Da jene nicht an Ländergrenzen Halt machen, kann ein einzelner Mitgliedstaat seine Bürger nicht effektiv schützen. Gleiches gilt für die Gefahr, die von möglichen Atomreaktorkatastrophen ausgeht. Da ein geordneter europaweiter Atom-

46 Vgl. Armin von Bogdandy, a.a.O. (Fn. 13), S. 24 f.

47 Zur Ursache vgl. oben.

48 Ähnliche Bedenken äußert Armin von Bogdandy, a.a.O. (Fn. 13), S. 25.

49 Vgl. Arthur Benz / Jörg Broschek, a.a.O. (Fn. 40), S. 20; Maja Moller Sousa, a.a.O. (Fn. 40).

50 Vgl. Christoph Grabenwarter, a.a.O. (Fn. 15), S. 157. 
ausstieg nicht absehbar ist ${ }^{51}$, ist ein Schutz nur durch europaweit hohe Sicherheitsstandards, deren Einhaltung durch unabhängige Stellen gesichert wird, möglich.

Berücksichtigt werden muss auch die Geschwindigkeit, in der Bundesregierung und Bundestag derzeit europapolitisch handeln (müssen). Der Zeitdruck darf nicht dazu führen, dass die Bundesregierung auf europäischer Ebene Entscheidungen ohne die erforderliche Ermächtigung des Bundestages trifft und dann nachträglich die Genehmigung dafür einfordert. ${ }^{52}$ Es ist zwar nicht auszuschließen, dass der Erlass eines ordentlichen Parlamentsgesetzes in einigen Situationen, die sofortiges, entschlossenes Handeln der europäischen Länder erfordern, zu langwierig wäre. Davon abgesehen, dass das tatsächliche Vorliegen einer solchen Situation überprüft werden muss, dürfen „Ausnahmesituationen“ nicht zum Freibrief für die Regierung werden. Um dem entgegenzuwirken, bedarf es zwar keines umfassenden Weisungsrechts in EU-Angelegenheiten. Es wäre aber begrüßenswert, wenn der Bundestag Mechanismen schaffen würde, die eine effektive Nutzung seiner bestehenden Mitwirkungsrechte auch in „Ausnahmesituationen“ garantieren. Der tatsächliche Gebrauch des aufgezeigten Stellungnahmerechts wäre eine mögliche Handlungsoption. Eine schnellere Handlungsfähigkeit kann der Bundestag dabei über Art. 45 S. 2 GG herstellen. Danach kann er den Ausschuss für Angelegenheiten der EU ermächtigen, für ihn die Rechte nach Art. 23 GG gegenüber der Regierung wahrzunehmen. Auch die Schaffung eines „limitierten“ Weisungsrechts wäre denkbar, das nur die Situationen erfasst, in denen die Gefahr besteht, dass aufgrund von Eile auf europäischer Ebene der Bundestag um seine zwingenden Mitwirkungsrechte gebracht wird. Damit wären die Interessen des Bundestages an der Wahrung seiner Mitwirkungsrechte mit den Interessen der Regierung an der Wahrung exekutiver Eigenverantwortung ${ }^{53}$ schonend zum Ausgleich gebracht.

Ein Weisungsrecht in allen Angelegenheiten der EU wäre jedoch verfassungswidrig. Ihm steht Art. 23 III 1, 2 GG entgegen. Der Wortlaut „berücksichtigt bei seinen Verhandlungen“ lässt eine derart absolute Bindungswirkung der Stellungnahme nicht zu. Andernfalls hätte es in Art. 23 III 2 GG ,ist an die Stellungnahme bei seinen Verhandlungen gebunden“ heißen müssen. Zudem wäre vom Umfang her der Wortlaut des Art. 23 III 1 GG überspannt, der eine Stellungnahme zu „Rechtsetzungsakten“, an denen die Bundsregierung

51 Großbritannien, Frankreich, Polen und Tschechien wollen sich in ihren Stellungnahmen für den „EU-Energiefahrplan bis 2050“ für eine Gleichstellung der Atomenergie mit den erneuerbaren Energien einsetzen. Vgl. dazu den Antrag der SPD-Fraktion vom 9. Mai 2012, BT-Drs. 17/9554, http://dipbt.bundestag.de/dip21/btd/17/095/1709554.pdf (Abruf am 26. Mai 2012).

52 Das war bei Zusagen der Regierung zum Europäischen Stabilitätsmechanismus (ESM) der Fall. „Politisch brisant ist die Angelegenheit, weil Deutschland in Brüssel bereits versprochen hat, beim Euro-Rettungsfonds für einen Betrag von bis zu 168 Milliarden Euro zu bürgen und sich zusätzlich an der notwendigen Bareinlage mit 22 Milliarden Euro zu beteiligen. Die notwendige Ermächtigung für diese Zusagen hat der Bundestag allerdings noch gar nicht gegeben." Paul Katzenberger, Euro-Schirm: Bundestag fühlt sich übergangen - „Da grummelt es gewaltig“, in: Süddeutsche.de vom 13. April 2011, http://www.sueddeutsche.de/geld/euro-schirm-bundestag-fuehlt-sich-uebergangen-da-grummelt-es-gewaltig-1.1084585 (Abruf am 26. Mai 2012). Zudem wurde dem Bundestag aufgrund der schlechten Informationsverschaffung durch die Bundesregierung (vgl. ebenda) die Grundlage für eine Stellungnahme vorenthalten. Die Bundesregierung verletzte damit auch die sich aus $\$ 4$ EUZBBG ergebende Pflicht zur umfassenden und frühestmöglichen Unterrichtung.

53 Das BVerfG hat in der Lissabon-Entscheidung (siehe Fußnote 1) der Bundesregierung eine gewisse Integrationsverantwortung zuerkannt. 
mitwirkt, vorsieht. Auch bei einer sehr extensiven Auslegung kann dieser Wortlaut nicht alle Angelegenheiten der EU erfassen. Wie bereits festgestellt, kann er nicht einmal alle Vorhaben im Sinne von $\$ 3$ EUZBBG erfassen.

Es wäre sogar fraglich, ob ein derart umfangreiches Weisungsrecht durch eine Änderung des Art. 23 III GG erreichbar wäre. Das verfassungsändernde Gesetz wäre möglicherweise selbst wegen Verstoßes gegen Art. 79 III GG verfassungswidrig. Das Gesetz muss das in Art. 20 III GG niedergelegte Rechtsstaatsprinzip und die dort angelegte Gewaltenteilung beachten. Letztere erfordert zwar keine strikte Gewaltentrennung, ein unantastbarer Kernbereich muss jeder Gewalt indes verbleiben. ${ }^{54}$ Bei dem von der Fraktion Die Linke vorgeschlagenen Entwurf zur Änderung des Art. 23 III 2 GG $^{55}$ bliebe ein gewisser Bereich der EU-Angelegenheiten unangetastet. Das Weisungsrecht bezöge sich „nur“ auf die Mitwirkung an Rechtsetzungsakten (Art. 23 III 1 GG) und wäre somit nicht umfassend. Allerdings wäre, gerade bei einer extensiven Auslegung des Art. 23 III 1 GG, sehr zweifelhaft, ob bei dem der Regierung verbleibenden unantastbaren Bereich noch von einem „Kernbereich“ in EU-Angelegenheiten gesprochen werden kann. Bei einem umfassenden Weisungsrecht, wäre dies jedenfalls nicht mehr der Fall. Nun könnte man argumentieren, dass die Europapolitik nicht zum Kernbereich der deutschen Exekutive gehört. Mit Hinblick auf den zunehmenden Einfluss der EU auf die nationale Ebene und der heutigen immensen Bedeutung der Europapolitik vermag dieses Argument die erheblichen Zweifel an der Verfassungsmäßigkeit eines solchen Gesetzes nicht auszuräumen. Verbliebe noch, unter Berücksichtigung, dass auch andere Länder ein Weisungsrecht mit ihren Verfassungen in Einklang bringen konnten, ein Erklärungsversuch für dessen verfassungsrechtliche Rechtfertigung. Hierbei soll der Unterschied zu einem Ermächtigungsrecht hervorgehoben werden. Letzteres greift unmittelbar in die Kompetenzen der Regierung ein. Durch das Erfordernis einer jeweiligen Ermächtigung der Bundesregierung durch den Bundestag in EU-Angelegenheiten wird bereits per Gesetz unmittelbar in den Kernbereich exekutiver Eigenverantwortung eingegriffen, weil die Regierung ohne Ermächtigung schlichtweg handlungsunfähig wäre. Die Handlungsfähigkeit auf EU-Ebene wäre dann nur im Einzelfall per Ermächtigung herstellbar. Beim Weisungsrecht ist es genau umgekehrt. Hier kann die grundsätzliche Handlungsfähigkeit im Einzelfall beschränkt werden, und zu einem tatsächlichen Eingriff in den Kernbereich kann es erst bei sehr intensiver Nutzung kommen. Daher könnte sich das BVerfG auf den Standpunkt stellen, dass es nur bei entsprechender Anrufung darüber zu befinden habe, ob die tatsächliche Handhabung des Weisungsrechts die generelle Handlungsfähigkeit der Regierung auf EUEbene in Frage stelle. Bei einem dem anderen Verfassungsorgan gegenüber rücksichtsvollen Umgang mit dem Weisungsrecht dürfte der Kernbereich exekutiver Eigenverantwortung nicht beeinträchtigt sein. Dies dürfte zumindest für ein Weisungsrecht bezogen auf Rechtsetzungsakte gemäß Art. 23 III 2 GG gelten. Dem Bundestag darüber hinaus in Fällen, die keinen Bezug zu legislativen Kompetenzen aufweisen, ein Weisungsrecht einzuräumen, lässt sich schon mangels seines schützenswerten Interesses nicht rechtfertigen.

54 Bundesverfassungsgericht, Entscheidungen des Bundesverfassungsgerichts, 110. Bd., Tübingen 2005, S. 199, S. 214 f.

55 „Die Bundesregierung ist an diese Stellungnahmen des Bundestages gebunden.“ Vgl. Gesetzentwurf zur Änderung des Grundgesetzes, BT-Drs. 16/13928, S. 3. 


\section{Verteidigung des Stellungnahmerechts in der Praxis}

Stellungnahmen entfalten nicht nur politische Bindungswirkung. Einfache Stellungnahmen nach \$9 II EUZBBG legen die Ausgangsposition der Regierung für Verhandlungen im Rat fest, können diese aber nicht verpflichten, diese Position bis zur Schlussabstimmung beizubehalten. Gebunden ist die Regierung bei ihrer abschließenden Entscheidung allenfalls politisch. Rechtlich ist sie dies bei qualifizierten Stellungnahmen nach $\$ 9$ IV EUZBBG. Eine Abweichungsmöglichkeit besteht aus wichtigen außen- und integrationspolitischen Gründen. Das Vorliegen eines Abweichungsgrundes müsste, ebenso wie die Einlegung eines Parlamentsvorbehaltes, die Verschaffung der Gelegenheit zur Stellungnahme ${ }^{56}$ sowie die Unterrichtung durch die Regierung vom BVerfG in einem Organstreitverfahren ${ }^{57}$ überprüfbar sein. ${ }^{58}$ Fraglich ist nur, inwieweit der Bundesregierung beim Abweichungsgrund ein Beurteilungsspielraum zu geben ist. Da es sich um nicht voll justiziable Prognoseentscheidungen handeln kann ${ }^{59}$, wird sich das BVerfG insoweit wohl auf eine Willkürkontrolle beschränken. Durch die gegenüber anderen Mitgliedstaaten auf „wichtige“ Gründe verengte Abweichungsmöglichkeit besteht die Gefahr eines Abweichens nach Gutdünken der Regierung jedoch nicht. Ob der Abweichungsgrund ein „wichtiger“ ist, müsste gerichtlich voll überprüfbar sein. Bei der Frage inwiefern dieser tatsächlich tangiert wird, dürfte der Regierung unter Umständen ein gewisser Beurteilungsspielraum zustehen. Hat der Bundestag eine Stellungnahme nach $\$ 9$ IV EUZBBG abgegeben, sollte die Regierung jedoch, angesichts der Antragsbefugnis von Oppositionsfraktionen im Organstreitverfahren, gehalten sein, ihr Abweichungsrecht restriktiv anzuwenden und im Zweifel eher den Bundestag um eine Abänderung seiner Position zu bitten, soweit diese im Rat nicht mehrheitsfähig ist. Andernfalls riskiert sie die Feststellung der Verfassungswidrigkeit ihres Vorgehens durch das BVerfG gemäß $\$ 67$ BVerfGG. Auf den verabschiedeten Ratsbeschluss hätte diese Entscheidung aber keinen Einfluss. Um der Verbindlichkeit auf europäischer Ebene zuvorzukommen, könnte eine einstweilige Anordnung nach $\$ 32$ BVerrfGG in Betracht gezogen werden. Ein diesbezüglicher Antrag kann vor Einleitung des Hauptsacheverfahrens gestellt werden. ${ }^{60}$ Zudem besteht die Möglichkeit, dass das BVerfG von Amts wegen tätig wird. ${ }^{61}$

56 Im Fall der EU-VO 407/2010 erhielt der Bundestag diese Gelegenheit nicht, Sebastian Gröningvon Thüna, a.a.O. (Fn. 7), S. 332. Zur Problematik der Verabschiedung des Euro-Stabilisierungspaketes vom 7. bis 9. Mai 2010, ebenda, S. 331 f. Ähnliche Probleme traten im Zusammenhang mit dem ESM auf. Vgl. dazu Fußnote 52.

57 Art. 93 Nr. 1 GG, $\$ \$ 13$ Nr. 5, 63 ff. BVerfGG.

58 Das Vorliegen einer verfassungsrechtlichen Streitigkeit, scheint, obwohl es sich bei dem verfassungskonkretisierenden $\$ 9$ IV EUZBBG um materielles Verfassungsrecht handelt, nicht selbstverständlich zu sein, denn die stark eingeschränkte Abweichungsmöglichkeit und der Parlamentsvorbehalt müssten wohl gemäß Bundesverwaltungsgericht (vgl. NJW 1985, H. 18, S. 2344) ihre unmittelbare Grundlage in Art. 23 III 2 GG finden. Zu den Abgrenzungsversuchen des BVerwG, vgl. Klaus Rennert, in: Erich Eyermann (Hrsg.), Verwaltungsgerichtsordnung, München 2010, $\$ 40$, Rn. 19 f.

59 Die gerichtliche Überprüfbarkeit von Regierungshandeln ist heute anerkannt, vgl. Helge Sodan, in: ders. I Jan Ziekow (Hrsg.), Verwaltungsgerichtsordnung. Großkommentar, Baden-Baden 2010, $\$ 40$, Rn. 177 mit weiteren Nennungen auch zur älteren anderen Auffassung.

60 Vgl. Helge Sodan, a.a.O. (Fn. 15), S. 400; Bundesverfassungsgericht, Entscheidungen des Bundesverfassungsgerichts, Bd. 42, Tübingen 1977, S. 103, S. $119 \mathrm{f}$.

61 Vgl. ebenda. 
Ein Bedürfnis für ein allgemeines Weisungsrecht in EU-Angelegenheiten gegenüber der Regierung besteht nicht. Die vorhandene Mitwirkungskompetenz des Bundestages ist ausgewogen. Bei Vorhaben, die die Übertragung von Hoheitsrechten betreffen, ist seit Einführung des IntVG flächendeckend die zwingende Mitwirkung des Bundestages sichergestellt. Vorhaben des 9 IV EUZBBG haben ihre parlamentarische Legitimation über die Zustimmung zum europäischen Primärrecht bereits erhalten. Daher ist die Mitwirkung hier dispositiv. Dennoch haben die europäischen Rechtsetzungsakte eine erhöhte Bedeutung, die es rechtfertigt, der Stellungnahme des Bundestages eine weitreichende Verbindlichkeit zukommen zu lassen. Einfache Stellungnahmen nach $\$ 9$ II EUZBBG sollen die politische Kontrolle der Regierung in Angelegenheiten der EU ermöglichen. Schließlich wäre ein allgemeines Weisungsrecht mit dem Grundgesetz unvereinbar. Auch eine dahingehende Verfassungsänderung wäre aufgrund des gemäß Art. 79 III GG unveräußerlichen Gewaltenteilungsprinzips als Ausfluss des Rechtsstaatsprinzips (Art. 20 III GG) wohl nicht möglich. Denkbar wäre die Schaffung eines Weisungsrechts für Fälle der berechtigten Sorge des Bundestages, dass seine Mitwirkungsrechte übergangen werden (limitiertes Weisungsrecht).

Der teilweise berechtigten Befürchtung, dass die EU stillschweigend ihre Kompetenzen erweitert oder Materien regelt, die besser auf nationaler Ebene geregelt werden könnten, können die nationalen Parlamente durch die bereits vorhandene Subsidiaritätskontrolle (Subsidiaritätsrüge und -klage) ${ }^{62}$ begegnen. Ein umfassendes Weisungsrecht ist dafür kein taugliches Mittel.

62 Vgl. $\$ \$ 11,12$ IntVG.

\section{Das Integrationsverantwortungsgesetz in der Praxis des Deutschen Bundestages}

Birgit Daiber

Das Integrationsverantwortungsgesetz ${ }^{1}$ wurde im September 2009 vom Deutschen Bundestag und vom Bundesrat beschlossen, um die Vorgaben des Bundesverfassungsgerichts in seinem Lissabon-Urteil vom 30. Juni desselben Jahres ${ }^{2}$ zu erfüllen. Ohne die dort verlangte Stärkung der Rechte beider Gesetzgebungsorgane in EU-Angelegenheiten hätte die Bundesrepublik Deutschland ihre Ratifikationsurkunde zum Vertrag von Lissabon nicht hinterlegen dürfen. ${ }^{3}$

1 BGBl. 2009 I, S. 3022 ff. Siehe dazu bereits Birgit Daiber, Die Umsetzung des Lissabon-Urteils des Bundesverfassungsgerichts durch Bundestag und Bundesrat, in: DÖV 2010, S. $293 \mathrm{ff}$.

2 BVerfGE 123, S. 267.

3 Ebenda, S. 270. 